\title{
PAM (Pregnancy Associated Malaria), Related Problems and Its Effective Treatments
}

\author{
Somia Gul, Tahira Khan, Shagufta Naz, Tayyaba Khan \\ Faculty of Pharmacy, Jinnah University for Women, Karachi, Pakistan \\ Email: drsomi1983@yahoo.com
}

Received 1 May 2014; revised 15 June 2014; accepted 25 July 2014

Copyright (C) 2014 by authors and OALib.

This work is licensed under the Creative Commons Attribution International License (CC BY). http://creativecommons.org/licenses/by/4.0/

(c) (i) Open Access

\begin{abstract}
Malaria is one of the leading causes of death and disease worldwide, especially in the developing world. Malaria which is transmitted by anopheles mosquitoes is a preventable and treatable infectious disease. It kills more than one million people each year, mostly in sub-Saharan Africa and other endemic areas. We have conducted a survey in different maternity homes to check the rate of occurrence of malaria during pregnancy and its complications in pregnant women, fetus and new born. Medical reports of 100 patients have been studied and analyzed for this purpose. Here we conclude that malaria can be prevented, diagnosed and treated with a combination of available tools and sustained financing in these pregnant ladies. A comprehensive approach consisting of protective nets, indoor spraying with insecticide, preventive treatment for pregnant women, diagnostic tests, effective drugs, education, research and advocacy is needed to combat malaria. The major complication that occurs in expectant female is anemia, and the major complication that occurs in newborns is cerebral hemorrhage. The best and advanced treatment used most commonly nowadays for malaria is Artesunate $\&$ its combinations.
\end{abstract}

\section{Keywords}

Malaria, PAM (Pregnancy Associated Malaria), Artesunate, Cerebral Hemorrhage

Subject Areas: Infectious Diseases, Pathology, Women's Health

\section{Introduction}

Malaria is a serious health problem in the developing world because of its high rates of morbidity and mortality [1]. Mostly children and pregnant women are immune-compromised individual so they have affinity for malaria [2]. Among all the malarial parasites, Plasmodium falciparum contributes to neurological complications, in- 
cluding agitation, psychosis, seizures, impaired consciousness and coma (cerebral malaria). The most severe one among all is cerebral malaria and the condition is associated with mortality of $15 \%-20 \%$, and a potential of developing neurocognitive sequelae [1].

There are almost 125 million women at risk of PAM every year; therefore diagnostic tools are needed for identification and treatment of malaria infection on time. According to study, nearly 50 million women face death due to PAM and an estimated amount of 200,000 children face fetal abnormalities and complications that lead to death due to their mothers' infection [3]. Pregnant women in malaria-endemic areas usually do not always receive the necessary treatment which contributes to severe adverse effects in mothers and infants [4]. The current tools are unable to detect a low parasitaemia as they remain too insensitive. The clinical data of available antimalarial drugs is insufficient because pregnant women cannot take part in clinical trials due to the chances of risk to both mother and the fetus, therefore a considerable effort, involving clinical trials, is needed to improve the diagnosis and case management of malaria during pregnancy for surveillance [5]. Such tools are needed to estimate disease burden and to make better public health policies [6]. Due to the weak immunity the women and the fetus are more prone to complication. Malaria in pregnancy cannot be prevented, but there is a need of accurate diagnosis and prompt treatment to stop dangerous symptomatic disease and to reduce its further effects on fetus [7].

Malaria infection is fatal during pregnancy and at the time of delivery. During a survey in Kenya, the prevalence of malaria was estimated and it appeared to be (85.7\%) in women having their 1st pregnancies and (51.7\%) in women having two or more previous pregnancies [8]. Pregnant women are at higher risk of malaria as compared to non-pregnant women. Maternal risk factors for PAM include low maternal age, low parity, and low gestational age. The complications of PAM include maternal anaemia, low birth weight (LBW), and preterm delivery [9].

Studies from sub-Saharan Africa showed that, approximately 25 million pregnant women every year are at risk of Plasmodium falciparum infection, and one in four women have placental infection at the time of delivery. $P$. falciparum infections during pregnancy in Africa rarely appear as fever and therefore remain undiagnosed and untreated. Analyses of intervention trials provide successful prevention of these infections and reduce the risk of severe maternal anemia by $38 \%$, low birth weight by $43 \%$, and per natal mortality by $27 \%$ among paucigravidae. An estimated 100,000 infant deaths occur in Africa each year due to LBW [10]. Plasmodium falciparum-infected red blood cells accumulate in the placenta through specific binding of pregnancy-associated parasite. Parasite accumulation, accompanied by an inflammatory infiltrate, disrupts the cytokine balance of pregnancy with the potential to cause placental damage and compromise foetal growth. Multigravid women develop immunity towards VAR2CSA-expressing parasites, and this antigen allows the parasite to attach to the cells of placenta [11]. The exposure of malaria in uterus results in neurological and cognitive deformities that affect the newborns performance and behavior. These effects are mediated by the initiation of neuroinflammation, dysregulation of neurovascular angiogenesis, and the disruption of normal synaptic pruning [12].

\section{Intermittent Preventive Treatment of Malaria for Pregnant Women (IPTp)}

Due to the presence of new organ i.e. Placenta parasite gets more sites to bind at, and this decreases the immunity of a pregnant women.

The major adverse effects of PAM appears on both the mother and the fetus which includes fetal loss, premature delivery, maternal anemia, intrauterine growth retardation, and low birth-weight infants delivery i.e. $(<2500$ g or $<5.5$ pounds), a risk factor of both maternal and infant death.

It is a problem for those women having their first and second pregnancies and for women who are HIV-positive.

These are the recommended practices for pregnant women:

- Use of medicated insecticide-treated bed nets (ITN)

- Intermittent preventive treatment (for women in high transmission areas)

- Effective case management (diagnosis and treatment of illness)

Women should be recommended iron/folate supplementation to protect them from anemia that occurs commonly among all pregnant women.

Administration of sulfadoxine-pyrimethamine at curative doses i.e. an effective anti-malarial drug; to all pregnant women whether or not they are infected with the malaria parasite. IPTp should be given at each routine antenatal care visit, from the second trimester. 
Folic acid supplementations are given to pregnant women to avoid neural tube defect in neonates but the effect of sulfadoxine-pyrimethamine is counteracted by high dose of folic acid. So it is preferable for women to take only the recommended $0.4 \mathrm{mg}$ daily dose of folic acid. In some countries, $5 \mathrm{mg}$ of folic acid are used, and there it is recommended to retain on folic acid supplementation for 2 weeks after taking IPTP with sulfadoxine-pyrimethamine for ensuring optimal efficacy [13].

\section{Results and Discussion}

The research was carried out in Karachi (Pakistan). A sample size of 100 pregnant women suffering from PAM was selected and a survey was conducted among them. The results of the survey showed that complications due to PAM cause severe harm to not only mother but to her fetus and lately to the new born. Mostly this malaria occurs due to $P$. falciparum. We have collected data to analyze that which complications are mostly occurring these days and what effective measures are taken by health care professionals to further avoid them.

The statistical data was collected through survey from gynecological wards and maternity homes located in Karachi, (Figure 1) shows that almost 35\% pregnant women develop a risk of anemia when they suffer from PAM. Different risk factors that aggravate complications in PAM and further contribute to the fetal and neonatal complexity are Primigravidae women, Pregnant women with one previous birth, pregnancy in young (adolescence) age and women in her second trimester of pregnancy also carries the highest risk of infection.

After analyzing the survey forms the results showed that most women were suffering from PAM in their second trimester that's why serious complications are reported as Figure 1 shows, 35\% women suffered from anemia, while $16 \%$ suffered from miscarriages and $13 \%$ from peurperal sepsis i.e. septicemia, other $10 \%$ from liver failure and $9 \%$ of hemorrhage and hypoglycemia and remaining 5\% from brain disorders and only $3 \%$ women were provided proper treatment (shown in Table 1) and hence no complication was seen in them.

Figure 2 shows that ACTs combination is used as the preferable treatment these days. Chloroquine is not used for falciparum malaria (plasmodium strains) because falciparum is resistant to it, $8 \%$ of patients received it (Figure 3). The 2 combinations are made with clindamycin i.e. quinine + clindamycin and artesunate + clindamycin these combinations were prescribed to $15 \%$ \& $18 \%$ of patients respectively. parental artesunate is usually given in severe cases $16 \%$ parental artesunate indicates severe complicated cases of PAM. Intermittent preventive therapy (IPT) with the malaria drug Sulfadoxine/pyramethamine is given to $13 \%$ patients, whether they are suffering from PAM or not, it reduces the risk of placenta malaria by 52 percents and it is efficacious for placental infections and severe maternal anemia.

Doxycycline, primaquine and all other tetracyclins are not recommended during pregnancy. Multiple drug treatment protocols are useful in treatment of the drug resistant plasmodium strain. Oral artemether is an alternative drug for the multiple drug resistant falciparum malaria. According to the World Health Organization (WHO), artemether is not recommended in the first trimester of pregnancy but must not be withheld in cases where it can save the life of the mother.

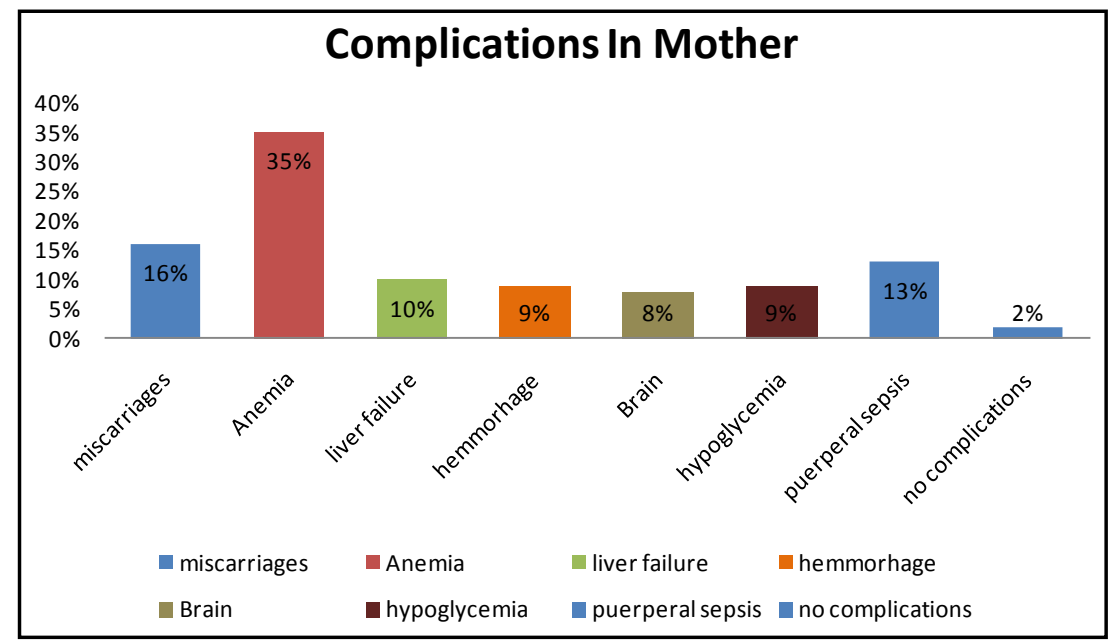

Figure 1. Complication in mother due to PAM. 


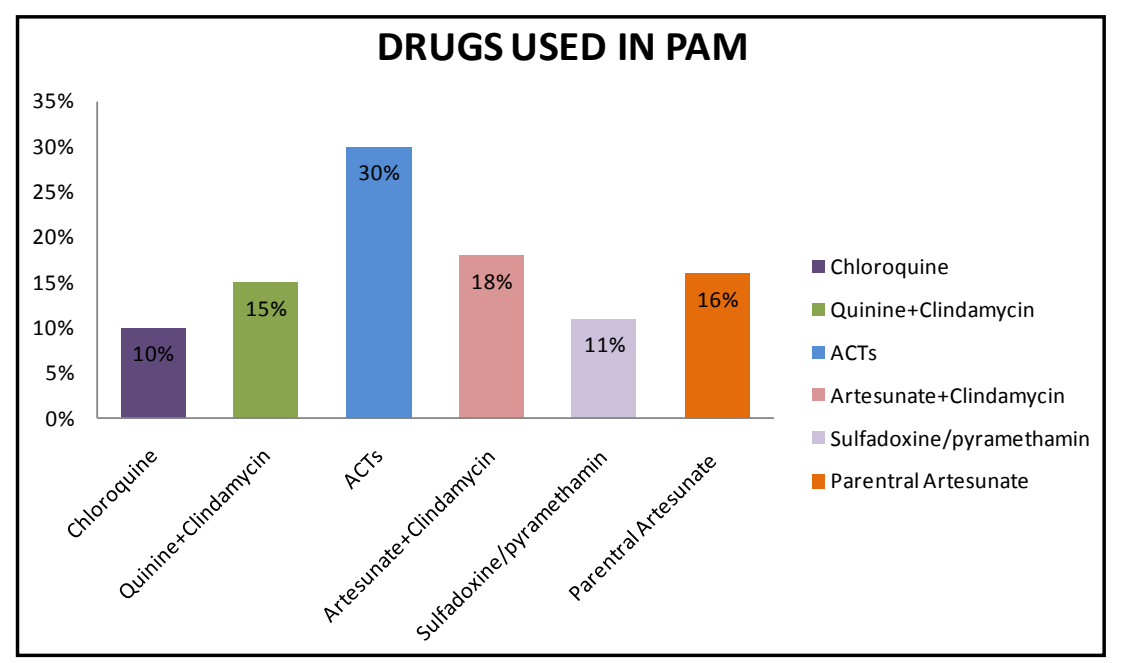

Figure 2. Drugs use in PAM (Pregnancy Associated Malaria).

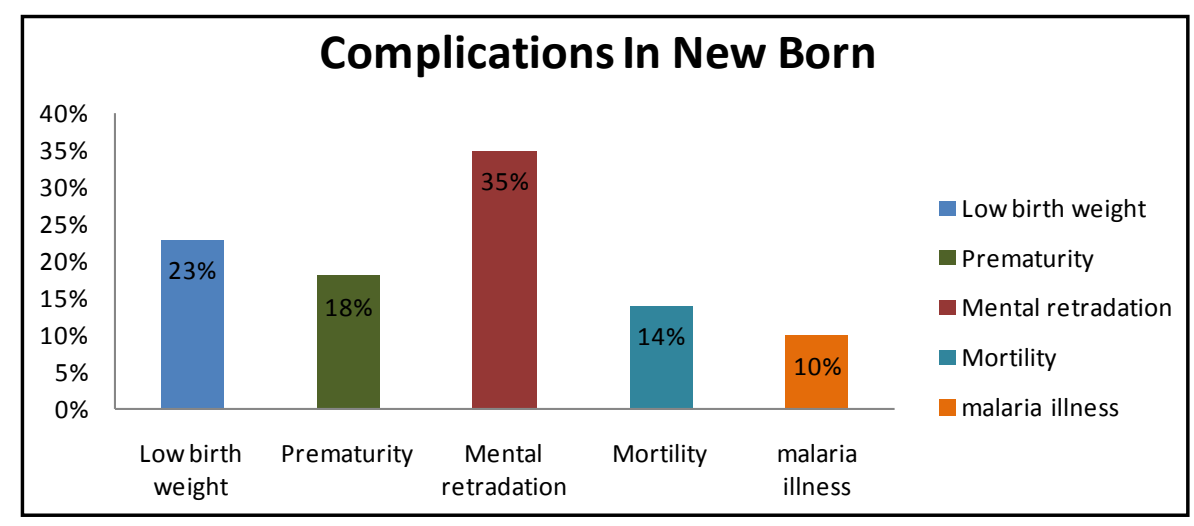

Figure 3. Complications commonly observed in new born due to PAM.

Table 1. Treatment of malaria in pregnancy.

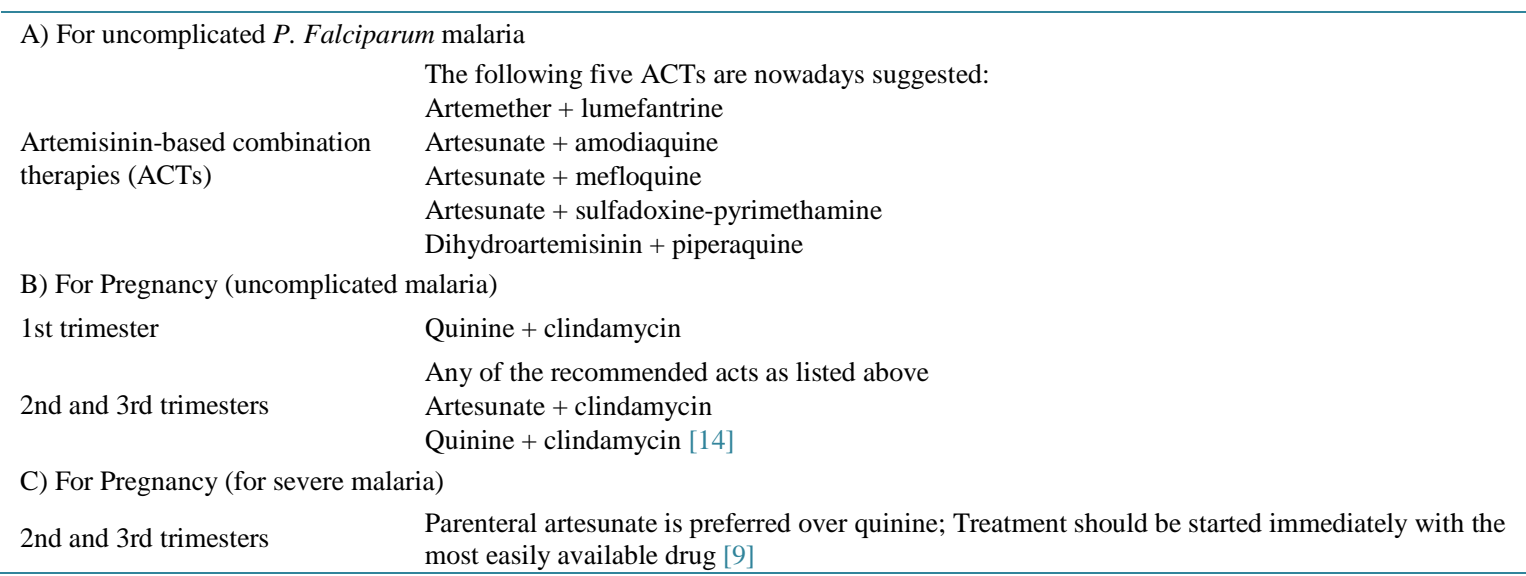

Figure 3 indicates that $60 \%$ fetus are aborted, $15 \%$ of them suffer from different congenital infections, $15 \%$ of still births occur due to PAM. Figure 4 indicates that almost 35\% newborns suffer from cerebral hemorrhage if their mothers previously suffered from PAM, other 23\% have low birth weight, 18\% newborns were premature at the time of delivery, $14 \%$ was mortality rate among newborns and $10 \%$ suffered from malaria illness as shown in Table 2. 
Malaria can be prevented, diagnosed and treated with a combination of available tools i.e. effective drugs, IPTp, protective nets, indoor spraying with insecticide, diagnostic tests, education, research and advocacy is needed to combat malaria.

Women should also be recommended iron/folate supplementation to protect them from anemia that occurs commonly among all pregnant women.

\section{Conclusion}

From the above conducted survey based research work, we conclude that malaria in pregnancy is causing disastrous complications in pregnant women as shown in Table 3, newborns and fetus. Intermittent preventive

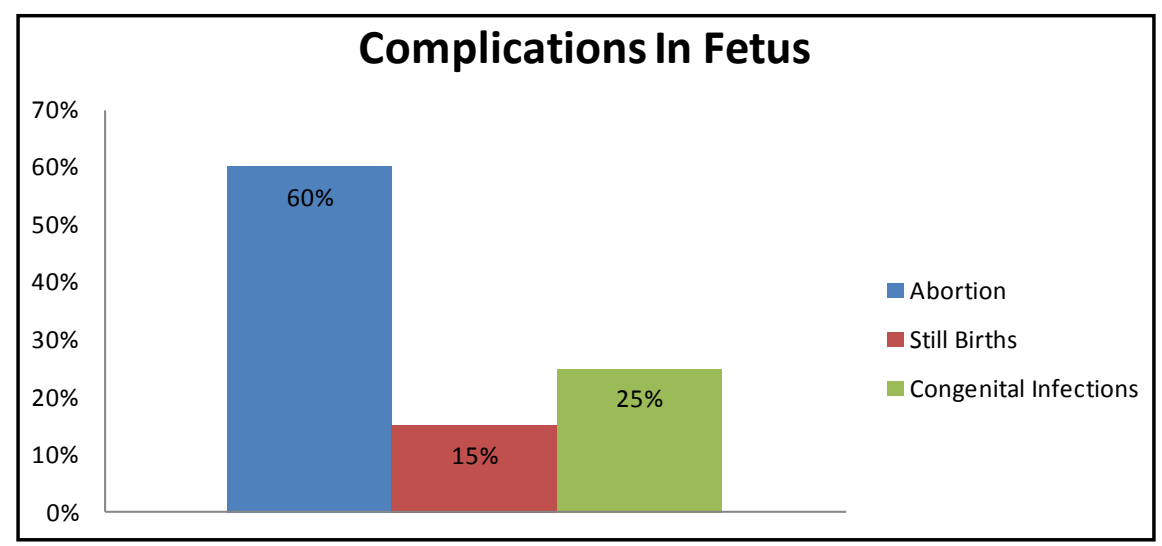

Figure 4. Complication in fetus due to PAM.

Table 2. Demographic data.

\begin{tabular}{|c|c|c|c|c|c|c|}
\hline \multicolumn{7}{|c|}{ COMPLICATIONS IN NEW BORN } \\
\hline \multicolumn{2}{|c|}{ Low birth weight } & Prematurity & Mental retardation & Mortality & \multicolumn{2}{|c|}{ Malaria illness } \\
\hline \multicolumn{2}{|c|}{$23 \%$} & $18 \%$ & $35 \%$ & $14 \%$ & \multicolumn{2}{|c|}{$10 \%$} \\
\hline \multicolumn{7}{|c|}{ DRUGS USED IN PAM } \\
\hline ACTs & Chloroquine & $\begin{array}{l}\text { Quinine + } \\
\text { clindamycin }\end{array}$ & $\begin{array}{l}\text { Artesunate + } \\
\text { clindamycin }\end{array}$ & $\begin{array}{c}\text { Parental } \\
\text { artesunate }\end{array}$ & \multicolumn{2}{|c|}{ Sulfadoxine/pyremethamine } \\
\hline $30 \%$ & $8 \%$ & $15 \%$ & $18 \%$ & $16 \%$ & \multicolumn{2}{|c|}{$13 \%$} \\
\hline \multicolumn{7}{|c|}{ COMPLICATIONS IN MOTHER } \\
\hline Miscarriages & Anemia & Liver failure & $\begin{array}{c}\text { Brain } \\
\text { disorder }\end{array}$ & hypoglycemia & $\begin{array}{l}\text { Puerperal } \\
\text { sepsis }\end{array}$ & $\begin{array}{c}\text { No } \\
\text { complications }\end{array}$ \\
\hline $16 \%$ & $35 \%$ & $10 \%$ & $5 \%$ & $9 \%$ & $13 \%$ & $3 \%$ \\
\hline \multicolumn{7}{|c|}{ COMPLICATIONS IN FETUS } \\
\hline \multicolumn{2}{|c|}{ Abortion } & \multicolumn{2}{|c|}{ Still Births } & \multicolumn{3}{|c|}{ Congenital Infection } \\
\hline \multicolumn{2}{|c|}{$60 \%$} & \multicolumn{2}{|r|}{$15 \%$} & \multicolumn{3}{|c|}{$25 \%$} \\
\hline
\end{tabular}

Table 3. Malaria in pregnancy: Double trouble.

\begin{tabular}{ll}
\hline More frequent & $\begin{array}{l}\text { Malaria is more usual in pregnancy as compared to the non pregnant, it is due to the suppression \& loss of } \\
\text { acquired immunity. } \\
\text { More aberrant }\end{array}$ \\
$\begin{array}{l}\text { In pregnancy, malaria is non confirming in presentation, it is due to the changes of hormones and immunity } \\
\text { during pregnancy. } \\
\text { Thore unrelenting }\end{array}$ & $\begin{array}{l}\text { The parasitemia is } 10 \text { times higher and as a result, all complications of falciparum malaria occurs. } \\
\text { non-pregnant population (6.5\%). }\end{array}$ \\
More virulent & $\begin{array}{l}\text { Some anti malarials are contra indicated in pregnancy like tetracyclins and some may cause severe adverse } \\
\text { effects. Therefore in cases of severe P. Falciparum malaria the treatment may become difficult. }\end{array}$ \\
Selective treatment & $\begin{array}{l}\text { Due to the various physiological changes of pregnancy management of complications in PAM is difficult. } \\
\text { Fluid intake, temperature control, etc. should be managed. The decisions regarding induction of labour may be } \\
\text { difficult and complex. Fetal loss, IUGR, and premature labour are very common [15]. }\end{array}$ \\
Some other & problems
\end{tabular}


treatment, insecticide treated bed nets and proper assistance and medications may contribute to avoiding such complications in expectant women. The major complications that occur in expectant female are anemia, and the major complication that occurs in newborns is cerebral hemorrhage. The best and advanced treatment used most commonly nowadays for malaria is artesunate and its combinations.

\section{References}

[1] Mishra, S.K. and Newton, C.R. (2009) Diagnosis and Management of the Neurological Complications of Falciparum Malaria. Nature Reviews Neurology, 5, 189-198.

[2] Schantz-Dunn, J. and Nour, N.M. (2009) Malaria and Pregnancy: A Global Health Perspective. Reviews in Obstetrics and Gynecology, 2, 186-192.

[3] Centers for Disease Control and Prevention (2010) The Rapid Assessment of the Burden of Malaria during Pregnancy: A Toolkit.

[4] Sangaré, L.R. and Stergachis, A. (2010) Determinants of Use of Intermittent Preventive Treatment of Malaria in Pregnancy: Jinja, Uganda. PLoS One, 5.

[5] Nosten, F., McGready, R. and Mutabingwa, T. (2007) Case Management of Malaria in Pregnancy. The Lancet Infectious Diseases, 7, 118-125.

[6] Conroy, A.L., McDonald, C.R. and Kain, K.C. (2012) Malaria in Pregnancy: Diagnosing Infection and Identifying Fetal Risk. Expert Review of Anti-Infective Therapy, 10, 1331-1342.

[7] Rijken, M.J., McGready, R., Boel, M.E., et al. (2012) Malaria in Pregnancy in the Asia-Pacific Region. The Lancet Infectious Diseases, 12, 75-88.

[8] Brabin, B.J. (1983) An Analysis of Malaria in Pregnancy in Africa. Bull World Health Organ, 61, 1005-1016.

[9] Takem, E.N. and D’Alessandro, U. (2013) Malaria in Pregnancy. Mediterranean Journal of Hematology and Infectious Diseases, 5.

[10] Desai, M., ter Kuile, F.O. and Nosten, F. (2007) Epidemiology and Burden of Malaria in Pregnancy. The Lancet Infectious Diseases, 7, 93-104.

[11] Kane, E.G. and Taylor-Robinson, A.W. (2011) Prospects and Pitfalls of Pregnancy-Associated Malaria Vaccination Based on the Natural Immune Response to Plasmodium falciparum VAR2CSA-Expressing Parasites. Malaria Research and Treatment, 2011, $21 \mathrm{p}$.

[12] McDonald, C.R., Elphinstone, R.E. and Kain, K.C. (2013) The Impact of Placental Malaria on Neurodevelopment of Exposed Infants: A Role for the Complement System? Trends in Parasitology, 29, 213-219.

[13] Centers for Disease Control and Prevention (2012) Intermittent Preventive Treatment of Malaria for Pregnant Women (IPTp).

[14] (2011) Global Fund Proposal Development: WHO Policy Brief on Malaria—WHO/GMP.

[15] http://www.malariasite.com/malaria/Pregnancy.htm 